\title{
Revised and updated catalogue of Byurakan-IRAS stars ${ }^{\star}$
}

\author{
A. M. Mickaelian and K. S. Gigoyan
}

\begin{abstract}
Byurakan Astrophysical Observatory and Isaac Newton Institute of Chile, Armenian branch, Byurakan 378433, Aragatzotn province, Armenia

e-mail: aregmick@apaven.am; kgigoyan@bao.sci.am
\end{abstract}

Received 14 January 2006 / Accepted 11 April 2006

ABSTRACT

\begin{abstract}
Context. Five lists of late-type stars were published in Astrophysics in 1997-2001, found in the First Byurakan Survey low-dispersion spectroscopic plates as optical identifications of unidentified IRAS sources. These identifications were carried out in the region with $\delta>+61^{\circ}$ and $|b|>15^{\circ}$ with a surface of $1504 \mathrm{deg}^{2}$. As a result, the catalogue of the Byurakan-IRAS Stars (BIS) was compiled. Its preliminary version has been available at the CDS since 2003 .

Aims. We revised and updated the BIS catalogue with the new data from recently published optical and infrared catalogues to give access to all available data and make further comparative studies of the properties of these objects possible.

Methods. We made cross-correlations of the BIS catalogue with the MAPS, USNO-B1.0, and 2MASS, added updated SIMBAD data for the BIS objects, and provided accurate DSS1 and DSS2 positions and revised photometry. We also checked the objects for proper motion and variability. A refined classification from low-dispersion spectra in the Digitized First Byurakan Survey (DFBS) was carried out.

Results. The revised and updated catalogue of 276 Byurakan-IRAS stars (BIS) is presented.

Conclusions. The BIS catalogue can be used to study a complete sample of IRAS-selected stars and to investigate individual objects.
\end{abstract}

Key words. catalogs - surveys - stars: carbon - infrared: stars

\section{Introduction}

In 1983, the Infrared Astronomical Satellite (IRAS) surveyed about $96 \%$ of the sky in bands centered at $12,25,60$, and $100 \mu$. More than 245000 point sources were detected and their fluxes and positions listed in the IRAS Point Source Catalogue (IRAS 1988). Sixty-six percent out of 245000 entries are stellar objects, and some 81000 objects have circumstellar shells (Van der Veen \& Habing 1988). Fainter sources are listed in the IRAS Faint Source catalogue (IRAS FSC) (IRAS 1990); however, to avoid the overcrowding of objects at low galactic latitudes, it includes objects outside of the Galactic plane $\left(|b|>10^{\circ}\right)$, so it has mostly extragalactic ones.

A large number of entries in IRAS PSC are not associated with objects in any of the available catalogues. In addition to photometric data (given in PSC), IRAS provides low-resolution spectra (LRS) from about 8 to $23 \mu$ using a slitless spectrometer. The spectra were observed in two sections: a blue section from 8 to $15 \mu$ with resolution $\lambda / \Delta \lambda \sim 40$ and a red section from 13 to $23 \mu$ with resolution $\sim 20$. This range $(8-23 \mu)$ is particularly important for the study of circumstellar shells since it contains the dust-grain signatures of both silicate dust around $10 \mu$ and $18 \mu$ and silicate carbide (SiC) dust around $11.3 \mu$. The IRAS LRS spectra have been divided into ten major classes determined by the shape of spectra (Olnon \& Raimond 1986; Kwok et al. 1997). This atlas contains the average spectra of 5425 objects. However, these are only a small part of the full set of observations. The

* Electronic BIS catalog (Table 1) is only available in electronic form at the CDS via anonymous ftp to

cdsarc.u-strasbg.fr $(130.79 .128 .5)$ or via

http://cdsweb.u-strasbg.fr/cgi-bin/qcat?]/A+A/455/765 database of all spectra during the IRAS mission contains 170000 individual scans for a total of $\sim 50000$ sources.

Because only about 70000 of the sources are associated with objects in astronomical catalogues, more identifications are required in order to realize and develop the IRAS potential. A lot of works have been devoted to the identification of IRAS point sources. Typically, the identifications have two criteria. The first is the coincidence with the IRAS position, and the second criterion is then one of the physical features of the objects (Jiang et al. 1996; Lyenger et al. 1989).

It has been shown that the positions of objects on the IRAS color-color diagrams can be used to characterize some of their fundamental physical parameters, such as the spectral type, the $\mathrm{C} / \mathrm{O}$ abundance ratio, or the presence of a circumstellar dust shell. The IRAS two color-color diagrams are primarily used from Van der Veen \& Habing (1988) to statistically consider the location of various type objects, oxygen-rich, carbon-rich, masslosing AGB long-period variables, planetary nebulae, among others, as well as for M and C (carbon) stars (Cohen et al. 1989; Walker \& Cohen 1998).

Many objects are classified based on their LRS spectra (Volk \& Cohen 1989; Little-Marenin et al. 1987; Volk et al. 1991; Kwok et al. 1997), and 11224 sources are listed in the last paper with their categorization into astrophysical classes, based on the presence of emission and absorption features and on the shape of the continuum. The IRAS color indices in combination with near-infrared $J H K L M$ photometry are used to select $\mathrm{M}$ - and C-type stars among the unassociated sources according to their color properties (Guglielmo et al. 1993, 1997, 1998). Optical identifications are provided for 102 color-selected IRAS PSC sources in the outer disk of our Galaxy (Jiang et al. 1996). 


\section{The Byurakan-IRAS stars}

Almost all previous IRAS identifications have been done by a pre-selection of sources, depending on whether the main task was to find stars or galaxies. This was done either by galactic latitudes or by IRAS colors, thereby distinguishing the stellar and extragalactic candidates. An optical identification program for the IRAS PSC was conducted in the Byurakan Astrophysical Observatory (BAO) in 1995 (Mickaelian 1995) using the First Byurakan Survey (FBS) low-dispersion spectra (Markarian et al. 1989). The area was defined by those FBS at $\delta>61^{\circ}$ having fields with galactic latitudes $|b|>15^{\circ}$, and the total surface was $1504 \mathrm{deg}^{2}$. The FBS low-dispersion spectra have an actual resolution of about 50A at $\mathrm{H} \gamma$ (corresponding to the photographic grains of the emulsion) and allow stars of different spectral types to be distinguished by their SED, although the lines themselves are typically not seen (beside the broad ones and spectral bands typical of late-type stars).

This program had a different approach from other similar works, as it aimed to identify all point sources without discrimination against their IR colors. No pre-selection was made to study the whole content of the IRAS PSC objectively. The galactic and extragalactic objects were distinguished later and two samples created: Byurakan-IRAS Galaxies (BIG), and Byurakan-IRAS Stars (BIS). In addition, the FBS lowdispersion spectra have significant advantages in identification of the nature of objects compared to mere cross-identifications with known catalogues or even to selection of objects by their colors, thereby leading to more efficiently revealing the optical counterparts.

As a result, the BIS objects are the optical identifications for IRAS PSC sources by means of the FBS lowdispersion spectra, where those appeared to be stars. Five lists of BIS (Mickaelian 1997; Gigoyan \& Mickaelian 1999, 2000; Mickaelian \& Gigoyan 2000, 2001) and 10 lists of BIG objects (Mickaelian \& Sargsyan 2004, and references therein) were published; more BIG objects will be published. The first list contained both stars and galaxies (paper IRAS1), 4 others had consequent enumeration, though 3 of them contained galaxies and another one (paper IRAS5) stars. The BIS/BIG enumeration was introduced starting with the 6th paper. At that moment, 179 galaxies and 137 stars had already been published (objects \#\# 1-316). Thus we decided to continue the enumeration with BIG 180 and BIS 138 (papers BIS3, BIS4, and BIS5). To avoid any confusion in the future, we also call the previously published objects BIG (numbers 1-179) and BIS (numbers 1-137). To help the users easily find objects both by the published numbers and by those introduced later, we give both in the catalogue. The preliminary version of the BIS catalogue has been available at the CDS since 2003 (Mickaelian \& Gigoyan 2003).

We measured accurate optical positions (for two epochs), obtained reliable photometric data after cross-correlation with MAPS, USNO-B1.0, and 2MASS catalogues, analyzed some of their data, checked the objects for proper motions (PM), refined the classification using the Digitized First Byurakan Survey (DFBS, Mickaelian et al. 2005) spectra, updated the available data from SIMBAD, and finally prepared a new catalogue of BIS objects.

\section{New data for the BIS objects}

The new data for the BIS objects are accurate optical positions measured from the DSS, MAPS/USNO-B1.0 photometry, revealing the variability and proper motions for a number of

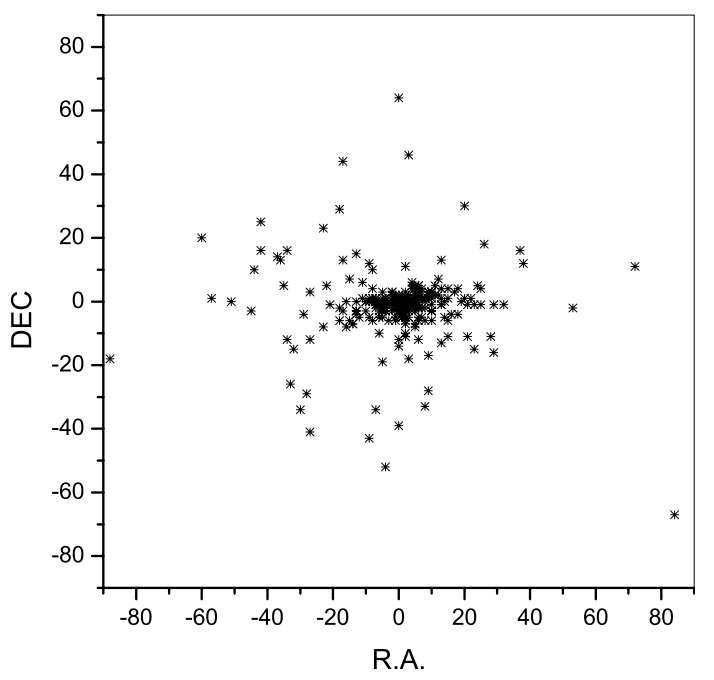

Fig. 1. DSS2r-IRAS positional differences for 276 identified BIS objects.

objects, and associations in the SIMBAD with previously known objects.

\subsection{Accurate optical positions}

For the new version of the catalogue, we measured the accurate DSS2 red optical positions using our experience with the FBS blue stellar objects (Mickaelian 2004). We obtained 0.5" accuracy in each coordinate, worse than the $0.33^{\prime \prime}$ given in the cited paper, as the BIS objects are brighter, and more accurate measurements are not possible. These accurate positions, as shown later, provided the possibility of correctly finding the corresponding associations in other known catalogues. The DSS1 positions were also measured (to reveal the proper motion stars), and after exclusion of the 3 PM stars, the rms correspondence between DSS1 and DSS2 was better than 1", making us feel confident that we have reliable positions.

A number of BIS objects had been known before (many bright stars, a number of FBS late-type stars); however, the identification with IRAS sources was not available either because of large errors in the old catalogues (as the associations in IRAS had been made using the positional cross-correlation) or PM for the bright stars and a shift between (typically) B1950 and the IRAS (1983) epochs. Other erroneous identifications had resulted from misidentification of 2 nearby objects, noncorrespondence of the given IRAS positional errors with the standard search box, etc. Because of PMs, the positions measured from DSS 1 and DSS 2 could be different. Some stars were shifted between DSS1 and IRAS epochs (25-35 years), compared to a 4-13 years shift between IRAS and DSS2, we used first the DSS2 position to achieve the best results.

To illustrate our results for optical identifications, we give the distribution of differences between the optical and IRAS positions in RA and Dec (Fig. 1). Sixty-three percent are within $10^{\prime \prime}, 87 \%$ within $30^{\prime \prime}$, and $98 \%$ within $60^{\prime \prime}$. Six objects have larger differences, but if taking the positions of their corresponding IRAS FSC sources, all their DSS2-IR positional differences are within $40^{\prime \prime}, 5$ of them even within $15^{\prime \prime}$. The reason is that the positions (and their corresponding errors) given in IRAS PSC were not accurate and were refined in IRAS FSC.

To better understand the identifications, we calculated the positional differences in the units of positional errors $(\sigma)$ given 


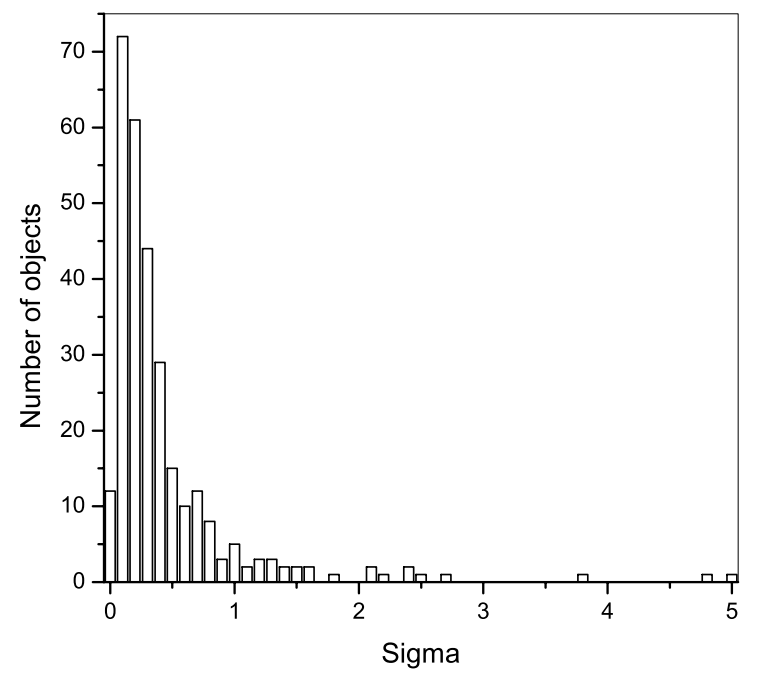

Fig. 2. Distribution of 276 BIS objects by the ratio of optical-IR positional differences and IRAS positional errors $(\sigma-s)$.

in the IRAS PSC catalogue. Figure 2 gives the distribution of our objects with respect to the fraction of $\sigma: 79 \%$ have been found within $0.5 \sigma, 92 \%$ within $1 \sigma, 97 \%$ within $2 \sigma$, and $99 \%$ are within $3 \sigma$. Three objects have larger differences between DSS2 and IRAS PSC $(3.8-5.0 \sigma)$. We consider these three cases as errors in the IRAS PSC catalogue, as their corresponding associations in IRAS FSC give only 3, 5, and 6 arcsec differences with the optical objects (all within $1 \sigma$ ).

After having the newly measured optical DSS2 (red) J2000 positions, a search for all identified objects was made in SIMBAD with a $60^{\prime \prime}$ search radius around these positions. At this point we could better judge if the association was genuine or false compared to associations with the IRAS positions. We found 227 associations in SIMBAD, including 1 A-type star, 1 F-type, 3 G-type stars (all these objects very bright), $28 \mathrm{~K}$-type, 163 M-type, 9 carbon stars, a planetary nebula, an X-ray source (without an optical name), and 20 other stars without classification. One $\mathrm{C}$ and $11 \mathrm{M}$ stars were found to be variables, including the semi-regular and Mira ones.

\subsection{MAPS/USNO-B1.0 photometry}

To have the best available photometric data for our objects, we cross-correlated the BIS list with the Minnesota Automated Plate Scanner (MAPS) catalogue (Cabanela et al. 2003), and the USNO-B1.0 (Monet et al. 2003). MAPS is based on measurements from the POSS1, while USNO- $B 1.0$ gives data from both POSS1 and POSS2. We carried out a statistical analysis of the photometric data provided by these two catalogues. When comparing their data from the POSS1 O and E images, we found a systematic shift. We used all 284 BIS objects (including some of those later rejected from the final sample) having all USNO- $B 1.0$ $B 1 / B 2 / R 1 / R 2$ data and 212 BIS objects having all MAPS (O and E) data.

First, we calculated the rms between the USNO $B 1$ and $B 2$ data $\left(1.13^{\mathrm{m}}\right)$ and between the $R 1$ and $R 2$ data $\left(0.45^{\mathrm{m}}\right)$. After elimination of 10 probable variables, these numbers were $1.00^{\mathrm{m}}$ and $0.24^{\mathrm{m}}$, respectively. We found a systematic shift of $0.88^{\mathrm{m}}$ between $B 1$ and $B 2$, and $0.08^{\mathrm{m}}$ between $R 1$ and $R 2$. After elimination of the 10 probable variables, the corresponding numbers were $0.91^{\mathrm{m}}$ and $0.07^{\mathrm{m}}$. Thus, most of the errors between $B 1$ and $B 2$ are due to the systematic shift, and there is a $\sim 0.1^{\mathrm{m}} \mathrm{rms}$ error in the USNO measurements, both for $B$ and $R$ magnitudes. Our conclusion is that $R$ magnitudes are more accurate and that one should be careful with $B$ magnitudes, otherwise a correction for the systematic shift is needed to calibrate them.

After elimination of the 10 probable variables, the rms between MAPS $O$ and USNO $B 1$ is 0.42 , between MAPS $O$ and USNO $B 2$ is 0.87 , and between MAPS $O$ and USNO $\langle B\rangle$ is 0.47 . It seems that MAPS magnitudes are calibrated closer to the USNO $B 1$, so that they can be used in combination to improve the photometry. The rms between MAPS E and USNO $R 1$ is 0.51 , between MAPS E and USNO $R 2$ is 0.51 , and $\langle R\rangle$ is 0.50 between MAPS E and USNO. Again, to improve the photometry, we found it better to average these data. In addition to the previous conclusion, we find that one should be especially careful with the USNO-B1.0 B2 magnitudes.

After elimination of the 10 probable variables, the systematic shift between MAPS O and USNO B1 is -0.18 , between MAPS $\mathrm{O}$ and USNO $B 2$ is 0.71 , and between MAPS $\mathrm{O}$ and USNO $\langle B\rangle$ 0.27 . The systematic shift between MAPS E and USNO $R 1$ is -0.32 , between MAPS E and USNO $R 2$ is -0.28 , and between MAPS E and USNO $\langle R\rangle-0.30$.

To reach the maximum possible photometric accuracy, we use the average between the MAPS $O$ and USNO $B 1$ as a $B$ magnitude, and when MAPS is absent (for some low galactic latitude fields), we use the corrected USNO $B 1(B=B 1-0.09)$. When USNO $B 1$ is absent, too, we use the corrected USNO $B 2$ $(B=B 2+0.79)$. For an $R$ magnitude, we take the average between MAPS E and USNO $R 1 / R 2$, and when MAPS is absent, we use the corrected average USNO R1/R2 $(R=\langle R\rangle-0.15)$. The values given in the catalogue are those calculated with the abovementioned principles.

\subsection{Variability}

To find out the variables in our sample, we used both the corrected USNO $B 1-B 2$ and $R 1-R 2$ differences, along with our estimates from the DSS1, DSS2, and DFBS images (as there are some accidental errors in the USNO catalogue too). The summarizing variability (given in the catalogue) is based on all these data.

To make a conclusion about the variability, we needed both USNO $B$ and $R$ differences between the two epochs (POSS 1 and POSS2) to be large enough and to have nearly the same value (to exclude accidental errors). We took an arbitrary limit for a confident variability (given as "var") when the average of $B 1-B 2$ and $R 1-R 2$ was $>0.80$ (well above the error values), and the errors between these numbers are smaller than half of their difference. Nine objects matched these criteria, and all were confirmed for variability from our inspection of the DSS1 and DSS2 images.

Probable variability (var:) was assigned in three cases: 1 ) to objects where the average of $B 1-B 2$ and $R 1-R 2$ was $>0.80$ but with large errors, 2) where the average of $B 1-B 2$ and $R 1-R 2$ $>0.50$ and the errors were smaller than the half of their difference, and 3$)$ where only one difference $(B 1-B 2$ or $R 1-R 2)$ was $>1.00$, and the other number was absent. All these suspected variables were checked by comparison of DSS1/DSS2/DFBS, and 9 out of 13 were confirmed, including 5 with large variability as seen from DSS1/DSS2.

We have 2 more objects (one genuine and one probable variable) found in addition to those obtained from the USNO-B1.0 analysis. Thus, 15 are listed as genuine variables (var) and 5 as probable ones (var:). Of course, there still may be other variables that were not revealed by our approaches. 
It is worth mentioning that out of 13 variables (Mira type, semi-regular, etc.) given in SIMBAD (see Sect. 3.5), only 5 were confirmed by our data. One more might be suspected as a variable, too, while 7 others do not show any variations by measurements from DSS1, DSS2, and DFBS. Since we find 10 more variables compared to SIMBAD, the two databases complement each other.

\subsection{Proper motions}

Proper motions (PM) are given in USNO-B1.0. However, based on our previous work (Mickaelian 2004), when a number of PM from the USNO were not confirmed, we decided to crosscheck them independently with our measurements from DSS1 and DSS2. We took the result as a real PM, if both data given in the USNO (in PM - RA and PM - Dec) match. According to the USNO catalogue, 11 objects in our list have $\mathrm{PM}>40 \mathrm{mas} / \mathrm{yr}$, including 5 with PM > 100 mas/yr. However, only 3 objects were confirmed to have PM: BIS 105 has PM = 152 mas/yr, BIS 225 has $\mathrm{PM}=187 \mathrm{mas} / \mathrm{yr}$, and BIS 251 has $\mathrm{PM}=397$ mas/yr. Our measurements give DSS2/DSS1 differences of 5.0", 8.2", and 23.2", corresponding to PM of $114,195,527$ mas/yr, respectively. The agreement between the USNO and our measurements is within $25 \%$.

Our measurements between DSS1 and DSS2 for all other objects were within $3^{\prime \prime}$. However, the USNO catalogue gives $\mathrm{PM}=245 \mathrm{mas} / \mathrm{yr}$ for BIS 161 and $\mathrm{PM}=228 \mathrm{mas} / \mathrm{yr}$ for BIS 186, while the DSS1 and DSS2 positions are identical for both these objects, so we rejected these two PM. Most probably, the problems with wrong PM data in USNO come from erroneous matching of the objects in plates from different epochs.

\subsection{SIMBAD associations}

The objects were checked for associations in SIMBAD. The search radius was $2^{\prime}$, and 226 associations were found. Almost all objects were found within 30". Only BIS 024 = CGCS 6083 was found at $114^{\prime \prime}$, but it is a correct association having a large positional error in SIMBAD. Stars from well-known HD, BD, GSC, and other catalogues were found, some having large positional uncertainties. Thirty FBS late-type stars were also found that were included in the FBS lists by Gigoyan et al. (2003).

Many of the BIS objects were thus known before; however, because of the positional errors in old catalogues, most of these objects could not be identified with IRAS sources by mere positional cross-correlation (as given in IRAS catalogues), which left them without a proper attention for studies of their IR properties. It is worth mentioning that the classification given in SIMBAD is mostly like " $\mathrm{K}$ " or " $\mathrm{M}$ ", or even just a "star" without a definite type or subtype, so in this cases we have estimated their spectral subtypes for the first time.

\section{The BIS catalogue}

We have revised and updated the BIS catalogue, including all available data from MAPS (O and E magnitudes and corresponding O-E color), USNO- $B 1.0(B 1, R 1, B 2, R 2$, and $I$ magnitudes, corresponding colors, and PM), 2MASS (2003) (JHK photometry), which allows us to follow their multiwavelength SEDs and to understand their nature, as well as we have carried out classification by the DFBS low-dispersion spectra and have added new SIMBAD data.
Two objects, BIS 44 and BIS 238 (found from different FBS zones), were found to be identical. After additional revision of our results, we found 11 objects had doubtful identifications (all from the 1st list and most probably faint galaxies, not stars). We rejected these objects in the current version of the BIS catalogue. Four misprints occurred in IRAS names in the original papers: published $06561+7539,15078+6156,16395+6439$, $17000+8235$ must be $06561+7359,15078+6155,16359+6439$, $17001+8235$, respectively. All these errors are corrected in this version as well.

The revised and updated BIS catalogue is given in Table 1. The catalogue consists of 276 objects and is given in order of right ascensions J2000. The contents of the BIS catalogue are as follows:

1. Byurakan-IRAS Star (BIS) number.

2. The original paper, where IRAS1 is Mickaelian (1997), IRAS5 is Gigoyan \& Mickaelian (1999), BIS3 is Mickaelian \& Gigoyan (2000), BIS4 is Gigoyan \& Mickaelian (2000), and BIS5 is Mickaelian \& Gigoyan (2001, see references).

3. Number of the object in the original paper.

4. IRAS Point Source catalogue (PSC) name.

5. IRAS PSC B1950 RA (hh:mm:ss.s).

6. IRAS PSC B1950 Dec (dd:mm:ss).

7. IRAS PSC positional uncertainty semi-major axis (SMJ) $(\operatorname{arcsec})$.

8. IRAS PSC positional uncertainty semi-minor axis (SMI) $(\operatorname{arcsec})$.

9. IRAS PSC uncertainty ellipse position angle (PA) (degrees).

10. IRAS PSC fluxes at $12,25,60$, and $100 \mu \mathrm{m}$ (Janskies).

11. IRAS PSC flux quality flags for $12,25,60$, and $100 \mu \mathrm{m}$.

12. IRAS FSC name (when available).

13. IRAS FSC B1950 RA (hh:mm:ss.s).

14. IRAS FSC B1950 Dec (dd:mm:ss).

15. IRAS FSC positional uncertainty SMJ (arcsec).

16. IRAS FSC positional uncertainty SMI $(\operatorname{arcsec})$.

17. IRAS FSC uncertainty ellipse PA (degrees).

18. Distance between the IRAS FSC and PSC positions ( $\operatorname{arcsec})$.

19. IRAS FSC fluxes at $12,25,60$, and $100 \mu \mathrm{m}$ (Janskies).

20. IRAS FSC flux quality flags for $12,25,60$, and $100 \mu \mathrm{m}$.

21. IRAS flux relative uncertainties in \% (FSC, when available).

22. IRAS (FSC, when available) [12-25] colors using the most common convention: [12]-[25] $=1.56-2.5 \log \left(F_{12} / F_{25}\right)$.

23. DSS1 J2000 RA (hh:mm:ss.ss), accurate to $1^{\prime \prime} \mathrm{rms}$.

24. DSS1 J2000 Dec (dd:mm:ss.s), accurate to $1^{\prime \prime}$ rms.

25. Distance between DSS1 and IRAS PSC positions in arcsec and in $\sigma-$ s.

26. Distance between DSS1 and IRAS FSC positions in arcsec and in $\sigma-\mathrm{s}$.

27. DSS2 red J2000 RA (hh:mm:ss.ss), accurate to $1^{\prime \prime}$ rms.

28. DSS2 red J2000 Dec (dd:mm:ss.s), accurate to $1^{\prime \prime}$ rms.

29. Distance between the DSS2 red and DSS1 positions ( $\operatorname{arcsec})$.

30. Galactic coordinates (l and b) (degrees).

31. Magnitudes and color indices as given in the BIS papers.

32. MAPS $\mathrm{O}$ and $\mathrm{E}$ magnitudes.

33. MAPS O-E color.

34. USNO-B1.0 $B 1, R 1$ (POSS1 epoch), and $B 2, R 2$ (POSS2 epoch) magnitudes.

35. Summarizing $B$ magnitude derived from MAPS and USNOB1.0 data.

36. Summarizing $R$ magnitude derived from MAPS and USNO$B 1.0$ data.

37. USNO-B1.0 I magnitude (POSS2 epoch IR). 
38. Summarizing $B-R$ color derived from MAPS and USNOB1.0 data.

39. Summarizing $R-I$ color derived from MAPS and USNO$B 1.0$ data.

40. 2MASS $J H K$ photometry and corresponding errors in each magnitude.

41. Corrected $B 1-B 2$ magnitude difference between POSS2 and POSS1 epochs.

42. Corrected $R 1-R 2$ magnitude difference between POSS 2 and POSS1 epochs.

43. Average between corrected $B 1-B 2$ and $R 1-R 2$ magnitude differences.

44. Variability flags ("var" or "var:").

45. Classification based on the DFBS low-dispersion spectra.

46. Associations taken from SIMBAD and with the FBS latetype stars.

47. SIMBAD classification.

48. SIMBAD $B$ and $V$ magnitudes.

49. Distance between the DSS1 and SIMBAD positions ( $\operatorname{arcsec})$.

50. Comments on the objects (binaries, etc.).

\section{Analysis of the BIS sample}

We made some analysis of the BIS sample, including that of the catalogue content and spectral classification, exploration of the color-magnitude diagram, discussion of some individual interesting objects, and analysis of the IR and multiwavelength properties of the BIS objects.

\subsection{The catalogue content and spectral types}

The revised spectral classification was made on the basis of the DFBS spectra. In the case where two or more DFBS plates were available in the field, they were all inspected for a better classification. The accuracy of the classification is about 2 subclasses, so that the classes are given as K8-K9, M 1-M 2, M 6-M 7, etc. (the accuracy of the FBS classification is restricted by its spectral resolution, about 50A at $\mathrm{H} \gamma$ ). In the BIS catalogue, there are one planetary nebula, 6 carbon stars $\mathrm{R}, \mathrm{N}$, or $\mathrm{C}$ (when the subtype is not available), 6 early $\mathrm{K}$ type (K0-K4), 36 late $\mathrm{K}$ type (K5-K9), 137 early M type (M0-M4), 89 late M type (M 5-M9) stars, and 1 unclassified object (strongly variable, absent in the FBS plates).

There are 205 objects associated in SIMBAD, where the spectral classification is given (many are taken from our BIS papers). However, for the majority (137), the classes are approximate (without a subclass) and of poor accuracy. In addition, 9 objects are listed as carbon stars, and 4 as possible carbon stars, but only 5 of them were confirmed by our spectra.

In Fig. 3, we give typical DFBS spectra for BIS objects from early to late types ( $\mathrm{K}-\mathrm{M}$ classes) and carbon stars $(\mathrm{R}$ and $\mathrm{N}$ classes). The K-M type and carbon stars obviously have different spectral energy distribution (SED) and absorption bands, which allows us to distinguish them easily (see their multiwavelength SEDs in Sect. 5.5). For the faint stars of the latest types, the spectra can be so short that only the red head is seen.

To check the reliability of our spectral classification (as well as our photometry derived from MAPS and USNO-B1.0 catalogues), we give the correspondence of our spectral classes in Table 2 to the corrected average $B-R$ and $R-I$ (MAPS/USNO$B 1.0), 2 \mathrm{MASS} J-H$ and $H-K$ colors, and IRAS [12-25] colors of the BIS objects; some subclasses (early ones) are grouped together because of the small number of objects. Note that $R-I$
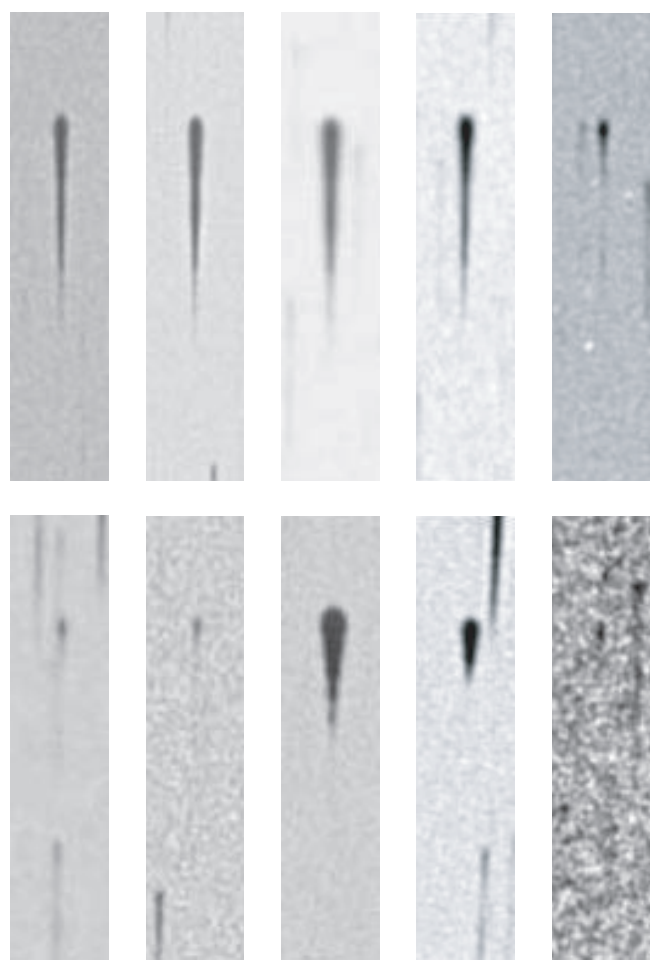

Fig. 3. Typical DFBS low-dispersion spectra for different types of red stars. First row: BIS 239 (K5-K6), BIS 137 (K8-K9), BIS 034 (M 1-M 2), BIS 029 (M 5-M 6), BIS 196 (M 7-M 8); second row: BIS 037 (M 8-M 9), BIS 173 (M 8-M 9), BIS 194 (R), BIS 184 (N), BIS $036(\mathrm{~N})$ (from left to right).

Table 2. Correspondence of the DFBS spectral classification of BIS objects to the corrected average $B-R$ and $R-I$ colors derived from MAPS and USNO-B1.0 catalogues, the 2MASS $J-H$ and $H-K$, and the IRAS [12-25] colors.

\begin{tabular}{lccccc}
\hline \hline DFBS types & $B-R$ & $R-I$ & $J-H$ & $H-K$ & {$[12-25]$} \\
\hline K0-K3 & 1.87 & 0.78 & 0.54 & 0.15 & 0.35 \\
K5-K8 & 2.60 & 0.70 & 0.77 & 0.24 & 0.20 \\
K8-K9 & 2.77 & 0.67 & 0.82 & 0.28 & 0.33 \\
K9-M0 & 2.73 & 0.70 & 0.83 & 0.28 & 0.27 \\
M0-M1 & 2.77 & 0.84 & 0.83 & 0.23 & 0.38 \\
M1-M2 & 2.78 & 0.77 & 0.84 & 0.28 & 0.22 \\
M2-M3 & 2.75 & 0.90 & 0.82 & 0.31 & 0.21 \\
M3-M4 & 2.83 & 1.01 & 0.85 & 0.33 & 0.30 \\
M4-M5 & 2.79 & 1.48 & 0.86 & 0.34 & 0.44 \\
M5-M6 & 2.96 & 1.60 & 0.89 & 0.35 & 0.43 \\
M6-M7 & 3.00 & 1.93 & 0.94 & 0.40 & 0.54 \\
M7-M8 & 3.11 & 1.90 & 0.92 & 0.43 & 0.57 \\
M8-M9 & 3.28 & 2.68 & 0.91 & 0.52 & 0.56 \\
\hline Carbon R & 3.69 & 1.96 & 1.02 & 0.49 & 0.34 \\
Carbon N & 7.40 & 2.36 & 1.51 & 1.10 & 0.38 \\
\hline
\end{tabular}

color is less accurate compared to B-R because there is the only measurement in $I$ band in USNO-B1.0. There is a reliable correspondence for the $B-R$ color (which was expected due to correction of the MAPS/USNO summarizing photometry), as well as for $R-I, R$-type carbon stars having approximately similar colors as the latest $\mathrm{M}$ type ones. The $J-H$ and $H-K$ colors (expecially the first one) are less sensitive to the spectral types, as is the IRAS [12-25] one. One can notice that our classification works better for later (M) types; note that the reason for larger errors at earlier types may also be the smaller number of such objects. 


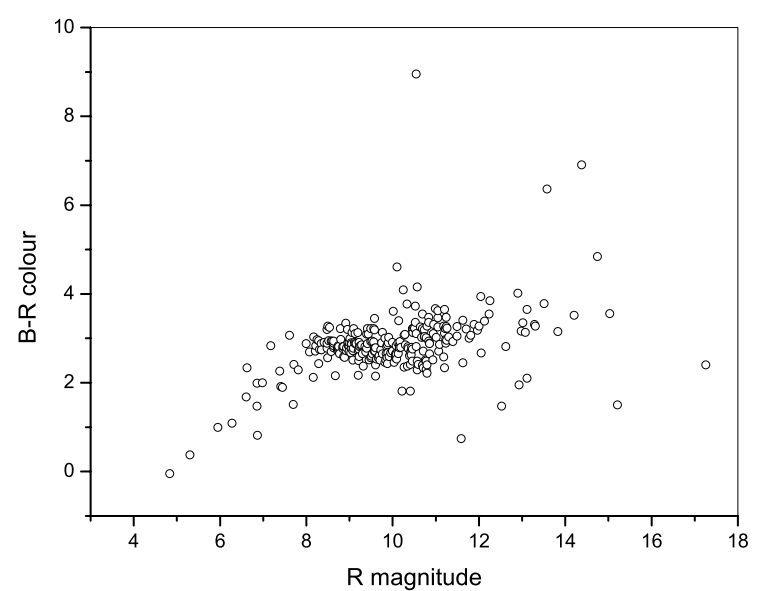

Fig. 4. $B-R$ color vs. $R$ magnitude diagram for 276 BIS objects.

\subsection{Color-magnitude diagram}

Figure 4 is the color-magnitude diagram for the BIS objects. It is obvious that (on average) the fainter the object, the redder it should be to appear in the IRAS catalogue. Thus we can determine the magnitude limits for stars with a given color to have an IR radiation detectable in IRAS; e.g., if the star has $B-R=+3$ (M 6-M 7 type), it should have at least $R=8^{\mathrm{m}}$ brightness to be detectable. Of course, there are stars with extended envelopes, which are strong IRAS emitters and which deviate from the above scheme.

We have studied all objects with other than the mean ratio of magnitudes and colors to find out some peculiarities in their properties. These are 4 very faint stars (variables, which could be much brighter during the IRAS observations, but are faint measured from the POSS images), namely BIS \#\# 10, 131, and 216 (and also BIS 222, which is not plotted in the figure because of the absence of its color), three extremely red stars, namely BIS \#\# 7, 36, and 184 (all N-type carbon stars; one more, BIS 197 having $B-R=4.61$, is a late $M$ type star), and 6 relatively blue stars distinguished in the lower part of the diagram, namely BIS \#\# 28 (PN), 60, 108, 165, 245, and 267, the last two objects being variables.

\subsection{Individual interesting objects}

Though almost all BIS objects are interesting because of their large IR fluxes, some of them are especially so due to their large variability, proper motion, rare spectral classes, etc. Here we give some details for the most interesting ones among these objects.

BIS $001=$ CGCS 6067. An $R$ star. In SIMBAD, the classification is $C$ without a subtype. It has large color indices: $B-R=3.78, R-I=3.47, R-K=7.28$.

$B I S 007=$ CGCS 6073. An N star, variable, with extremely red colors: $B-R=6.36, R-I=5.41, R-K=9.38$.

$B I S 010=$ CGCS 6074. A C star (without accurate classification). If judging from the different colors, this object should be of $\mathrm{N}$ type rather than $R$.

$B I S$ 028. PN. The only object in the sample that is relatively blue $(B-R=0.74)$ with $R=11.59$. Some details about this object are given in other sections as well.

BIS 036 = CGCS 6098. An N star. In SIMBAD, the classification is $\mathrm{C}$ without a subtype. It has extremely red colors: $B-R=6.91, R-K=6.13$.
BIS 090. An M 0-M 1 type star. The only X-ray (ROSAT) source in the sample: RX J0655.5+6431.

$B I S 105=\mathrm{HD}$ 86855. A K2-K3 type star that has a large proper motion in both RA (-96 mas/yr) and Dec (-118 mas/yr); total PM is $152 \mathrm{mas} / \mathrm{yr}$ according to USNO-B1.0, and $114 \mathrm{mas} / \mathrm{yr}$ according to our measurements $(\mathrm{PM}-\mathrm{RA}=$ $-43, \mathrm{PM}-\mathrm{Dec}=106)$.

BIS 138 = FBS 0350+694. An M 5-M 6 type star (M 3 according to SIMBAD) and one of the brightest IR sources in the sample $\left(F_{12}=25.01 \mathrm{Jy}, F_{25}=16.84 \mathrm{Jy}\right)$, also having an IRAS low-resolution spectrum with the LRS Atlas code 24 (Volk et al. 1991) and "E" (Kwok et al. 1997).

BIS $171=\mathrm{BD}+71$ 876. An M6-M 7 type star (M 6.5 according to SIMBAD) and one of the brightest IR sources in the sample $\left(F_{12}=11.72 \mathrm{Jy}, F_{25}=7.03 \mathrm{Jy}\right)$, also having an IRAS low-resolution spectrum with the LRS Atlas code "E" (Kwok et al. 1997).

BIS $176=\mathrm{KN}$ Cam $=$ FBS 0532+673. An M7-M 8 type star (M 6 according to SIMBAD). Mira type variable and one of the brightest IR sources in the sample $\left(F_{12}=10.03 \mathrm{Jy}, F_{25}=\right.$ $3.96 \mathrm{Jy})$, also having an IRAS low-resolution spectrum with the LRS Atlas code 14 (Volk et al. 1991) and "F" (Kwok et al. 1997).

BIS 184 = HP Cam = FBS 0601+675. An N star $(\mathrm{C}$ according to SIMBAD) that is strongly variable according to both MAPS/USNO photometry $(B 1-B 2=4.70, R 1-R 2=1.92)$ and our inspection of the DSS1/DSS2 images. Semi-regular variable according to SIMBAD. It has extremely red colors: $B-R=8.95$, $R-K=7.15$. One of the brightest IR sources in the sample $\left(F_{12}=25.26 \mathrm{Jy}, F_{25}=6.97 \mathrm{Jy}\right)$, also having an IRAS lowresolution spectrum with the LRS Atlas code 44 (Volk et al. 1991) and "C" (Kwok et al. 1997).

$B I S$ 194. An $\mathrm{R}$ star. Has large color indices $B-R=3.61$, and $R-K=$ 5.36. BIS $196=$ FBS 0749+654. An M7-M 8 type star. Strongly variable from both MAPS/USNO photometry $(B 1-B 2=-5.27, R 1-R 2=-0.40)$ and our inspection of the DSS1/DSS2 images.

$B I S$ 222. The only unclassified object (because of the absence of the FBS low-dispersion spectrum). Strongly variable; the object is present only in DSS1 red and has $R 1=17.41 \mathrm{ac}-$ cording to USNO-B1.0. The colors of this object are rather peculiar, probably due to its strong variability (no classification is appropriate).

BIS $225=$ HD 30097. An M 4-M 5 type star. It has a large proper motion in both RA (82 mas/yr) and Dec (-168 mas/yr); total PM is $187 \mathrm{mas} / \mathrm{yr}$ according to USNO-B1.0, and $195 \mathrm{mas} / \mathrm{yr}$ according to our measurements $(\mathrm{PM}-\mathrm{RA}=80$, $\mathrm{PM}-\mathrm{Dec}=-178)$, with excellent agreement between these two data $(4 \%)$.

BIS $243=$ YZ Cam. An M7-M8 type star that is strongly variable from both MAPS/USNO photometry $(B 1-B 2=-4.92$, $R 1-R 2=-2.02$ ) and our inspection of the DSS1/DSS2 images. It is a Mira type variable according to SIMBAD.

$B I S$ 245. An M 5-M 6 type star that is strongly variable from both MAPS/USNO photometry $(B 1-B 2=-1.94, R 1-R 2=$ -2.14 ) and our inspection of the DSS1/DSS2 images.

BIS $251=$ HD 62613. A bright, G9-K0 type star, with the largest proper motion among the objects in the sample. According to USNO-B1.0, PM-RA $=-388 \mathrm{mas} / \mathrm{yr}$, $\mathrm{PM}-\mathrm{Dec}=84 \mathrm{mas} / \mathrm{yr}$, total PM being $397 \mathrm{mas} / \mathrm{yr}$. Our measurements give $\mathrm{PM}-\mathrm{RA}=-507 \mathrm{mas} / \mathrm{yr}$ and $\mathrm{PM}-\mathrm{Dec}=$ $142 \mathrm{mas} / \mathrm{yr}$, total PM = $527 \mathrm{mas} / \mathrm{yr}$. The DSS2/DSS1 difference is $23.2^{\prime \prime}$ (44 years difference in observations). 


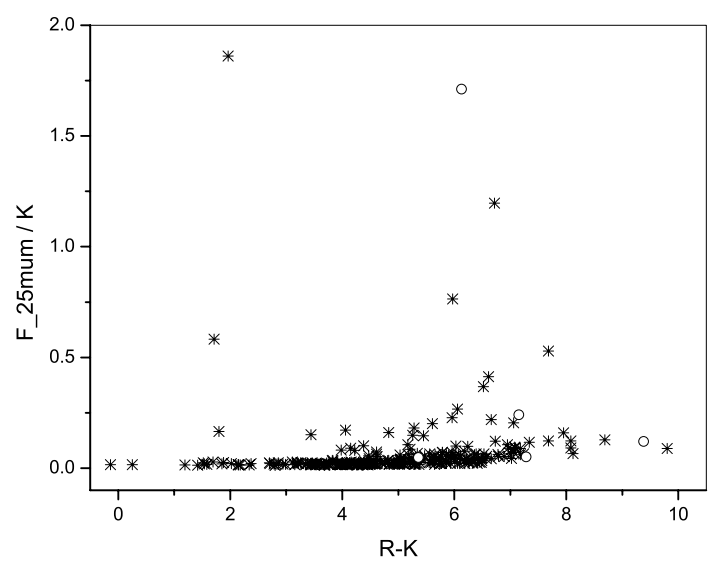

Fig. 5. The $F_{25} / K$ vs. $R-K$ color for 271 BIS objects. K-M stars are marked by asterisks, and open circles are for $\mathrm{C}$ stars. A number of objects are located well above the "main sequence" defined by the normal spectral energy distribution.

BIS 267. An M 3-M 4 type star that is strongly variable from both MAPS/USNO photometry $(B 1-B 2=-4.22, R 1-R 2=$ $-2.69)$ and our inspection of the DSS1/DSS2 images.

\subsection{Infrared properties of the BIS objects}

Most of the BIS objects are confidently detected (quality flags 2 and 3 ) at IRAS bands $12 \mu \mathrm{m}$ and $25 \mu \mathrm{m}$, both in PSC (274 objects at $12 \mu \mathrm{m}$, and 125 at $25 \mu \mathrm{m}$ out of 276) and FSC (268 objects at $12 \mu \mathrm{m}$, and 250 at $25 \mu \mathrm{m}$ out of 269). The $60 \mu \mathrm{m}$ and $100 \mu \mathrm{m}$ fluxes are available for only the brightest objects. It appears that the IRAS PSC and FSC fluxes at $12 \mu \mathrm{m}$ are more confident than those at $25 \mu \mathrm{m}$ : the average difference between these two catalogues' data at $12 \mu \mathrm{m}$ is $0.0326 \mathrm{Jy}$, while this difference for $25 \mu \mathrm{m}$ fluxes is $0.0953 \mathrm{Jy}$. However, when available, we accept the FSC values, which are more accurate.

The sample may be analyzed by the IR-optical flux ratios, as different objects are different in this sense. We built a few color-color diagrams to analyze the sample and discover peculiar objects.

We plot the $F_{25} / K$ vs. $R-K$ color in Fig. 5 for 271 BIS objects, except one absent in 2MASS, 3 with large IR-opt flux ratios (to analyse the details for other objects), and the abovementioned PN. This PN has a completely different IR-opt ratio; its $R-K$ color is -3.15 , and the $F_{25} / K$ ratio is 714.8. Three objects, namely BIS 10 (C star), BIS 131 (M 4-M 5), and BIS 222 (the object without a spectral classification), have extremely large IR fluxes $\left(F_{25} / K 95.6,34.1\right.$, and 6.7, respectively) for their optical and NIR (2MASS) magnitudes, which probably indicates the existence of powerful circumstellar shells, unless these objects are strongly variable (evidence of variability is for BIS 222 only) and the measurements are not for the same brightness phase.

Even though the $y$-scale in Fig. 5 is highly enlarged, there are still some objects with peculiar IR-opt flux ratios (compared to the vast majority with normal spectral energy distribution). These are BIS 60 (K0-K1), BIS 36 (N), BIS 20 (M6-M 7), BIS 2 (M 0-M 1), and BIS 100 (M4-M 5). As their spectral types are completely different and there is no evidence of variability, we assume they could be stars with large envelopes enhancing their IR fluxes.

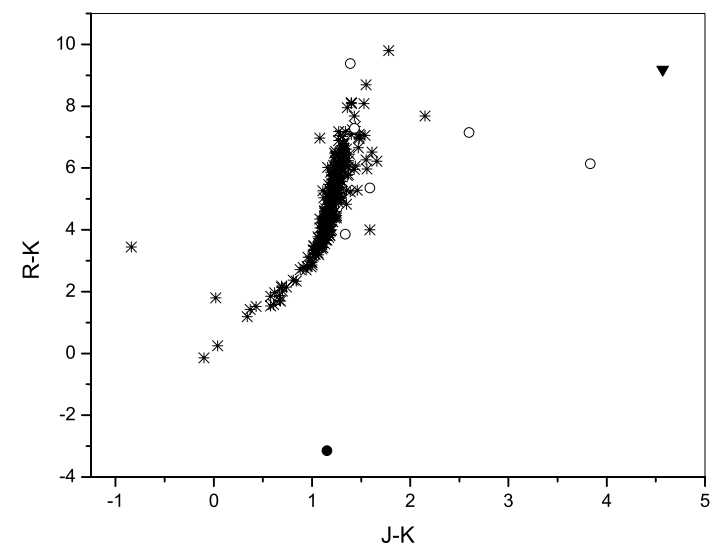

Fig. 6. The $R-K$ vs. $J-K$ color-color diagram for 275 BIS objects. $\mathrm{K}-\mathrm{M}$ stars are marked by asterisks, open circles are for $\mathrm{C}$ stars, the filled circle is for the PN, and the triangle is for the unclassified object. A number of objects are located outside the "main sequence" defined by the normal spectral energy distribution.
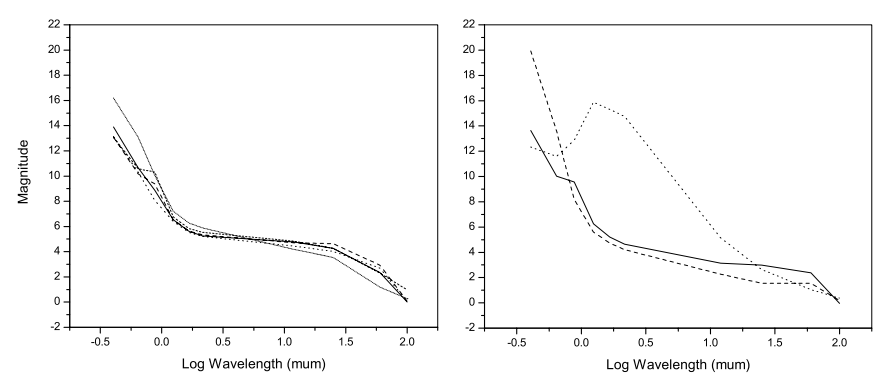

Fig. 7. Multiwavelength spectral energy distribution (SED) for different types of stars and planetary nebula. Left panel: G9-K0 (solid line), K4-K5 (dashed line), K9-M 0 (short dashed line), M4-M 5 (dotted line), M 8-M 9 (short dotted line). Right panel: R (solid line), N (dashed line), PN (dotted line).

We have calculated the $F_{12} / K$ ratios, too, and found that the PN BIS 28 also has an extraordinarily large $F_{12} / K$ ratio (301.8). And it is worth mentioning that the same three objects (BIS 10, BIS 131, and BIS 222) have extremely high $F_{12} / K$ ratios too. Another diagram using the optical and NIR fluxes, $R-K$ vs. $J-K$ is shown in Fig. 6. Again, besides the PN, two $\mathrm{N}$ type stars, and the unclassified object, there are a few other objects showing peculiar colors compared to the vast majority.

\subsection{Multiwavelength properties}

Using the optical $(B-R-I)$, NIR $(J-H-K)$, and MIR (12 and $25 \mu \mathrm{m}$; data at 60 and $100 \mu \mathrm{m}$ are inaccurate) magnitudes, we have constructed the multiwavelength SEDs for the BIS objects.

Figure 7 gives some examples of these SEDs for G9-K0, K4-K5, K9-M 0, M 4-M 5, M 8-M 9, R, and N type stars, as well as for the PN. The stars were selected with approximately similar magnitudes at IRAS $12 \mu \mathrm{m}$ to show the distribution at the same level. Except for the planetary nebula, all other objects have thermal SEDs with slight deviations. A break at the $I$ magnitude is noticeable for some objects, especially for the $R$ type carbon star. Such spectra may be useful for approximate classification of unclassified objects. 


\section{Summary}

Using our previous data on the BIS objects, we have revised and updated the BIS catalogue by adding presently available data from the DSS1/DSS2 (positions and proper motions), MAPS (optical photometry), USNO-B1.0 (optical photometry and proper motions), and 2MASS (NIR photometry) catalogues, by re-classifying the objects by means of the DFBS lowdispersion spectra, and by updating other available data from SIMBAD. Accurate optical positions were measured allowing a correct match with the known objects and exclusion of the previous erroneous associations.

We revealed a new PM and variable stars. A preliminary analysis of the sample for its contents and the optical and IR properties was carried out, while individual interesting objects were distinguished and described.

An analysis of the MAPS and USNO-B1.0 photometric data revealed a $0.91^{\mathrm{m}}$ systematic shift between the USNO measurements from the POSS1 and POSS2 plates for the $B$ magnitudes. Even though if the USNO $B$ magnitudes are corrected, there is still a systematic shift between the MAPS and USNO data: $-0.18^{\mathrm{m}}$ in $B$ and $-0.30^{\mathrm{m}}$ in $R$. A transformation formula was derived to reach the maximum available photometric accuracy $\left(0.1^{\mathrm{m}}\right)$ based on the MAPS and USNO catalogues.

The BIS sample is now being used to compile a total list of IRAS PSC sources with their optical identification and relevant data in the FBS area, which will be finished after inspection and correction of the previously known identifications.

Acknowledgements. The Digitized Sky Surveys were produced at the Space Telescope Science Institute under US Government grant NAG W-2166. The images of these surveys are based on photographic data obtained using the Oschin Schmidt Telescope on Palomar Mountain and the UK Schmidt Telescope. The plates were processed into the presently compressed digital form with the permission of these institutions. The National Geographic Society - Palomar Observatory Sky Atlas (POSS-I) was made by the California Institute of Technology with grants from the National Geographic Society.

The Second Palomar Observatory Sky Survey (POSS-II) was made by the California Institute of Technology with funds from the National Science Foundation, the National Geographic Society, the Sloan Foundation, the Samuel Oschin Foundation, and the Eastman Kodak Corporation. The Oschin Schmidt Telescope is operated by the California Institute of Technology and Palomar Observatory. The MAPS catalogue is supported by the National Science Foundation, the National Aeronautics and Space Administration, and the University of Minnesota, and is available at http://aps.umn.edu/.

This research made use of the SIMBAD database, operated at the CDS, Strasbourg, France, the USNO-B1.0 catalogue, supported by the United States
Naval Observatory, and Two Micron All-Sky Survey (2MASS), a joint project of the University of Massachusetts and the Infrared Processing and Analysis Center (IPAC, Caltech).

The authors are thankful to Dr. M. Cohen for a careful reading of the manuscript and valuable comments.

\section{References}

Cabanela, J. E., Humphreys, R. M., Aldering, G., et al. 2003, PASP, 115, 837 Cohen, M., Wainscoat, R. J., Walker, H. J., et al. 1989, AJ, 97, 1759

Cutri, R. M., Skrutskie, M. F., Van Dyk, S., et al. 2003, The 2MASS All-Sky Catalog. Final Release, University of Massachusetts and IPAC/California Institute of Technology

IRAS 1988, Joint IRAS Science Working Group. Infrared Astronomical Satellite Catalogs, The Point Source Catalog, Version 2.0, NASA RP-1190

Gigoyan, K. S., \& Mickaelian, A. M. 1999, Ap, 42, 37

Gigoyan, K. S., \& Mickaelian, A. M. 2000, Ap, 43, 262

Gigoyan, K. S., Abrahamyan, H. V., Azzopardi, M., et al. 2003, Ap, 46, 577

Guglielmo, F., Epchtein, N., \& Le Bertre, T., et al. 1993, A\&AS, 99, 31

Guglielmo, F., Epchtein, N., Arditti, F., \& Sevre, F. 1997, A\&AS, 122, 489

Guglielmo, F., Le Bertre, T., \& Epchtein, N. 1998, A\&A, 334, 609

Jiang, B. W., Deguchi, S., \& Nakada, Y. 1996, AJ, 111, 231

Lasker, B. M., Doggett, J., McLean, B., et al. 1996, ASP Conf. Ser., 101, 88

Little-Marenin, I. R., Ramsay, M. E., \& Stephenson, C. B., et al. 1987, AJ, 93, 663

Lyenga, K. V. K., Ghosh, S. K., Rengarajan, T. N., et al. 1989, A\&A, 221, 250

Kwok, S., Volk, K., \& Bidelman, W. P. 1997, ApJS, 112, 557

McGlynn, T., White, N. E., \& Scollick, K. 1994, ASP Conf. Ser., 61, 34

Markarian, B. E., Lipovetski, V. A., Stepanian, J. A., Erastova, L. K., \& Shapovalova, A. I. 1989, Commun. Special Astrophys. Obs., 62, 5

Mickaelian, A. M. 1995, Ap, 38, 349

Mickaelian, A. M. 1997, Ap, 40, 1

Mickaelian, A. M. 2004, A\&A, 426, 367

Mickaelian, A. M., \& Gigoyan, K. S. 2000, Ap, 43, 55

Mickaelian, A. M., \& Gigoyan, K. S. 2001, Ap, 44, 222

Mickaelian, A. M., \& Gigoyan, K. S. 2003, Byurakan-IRAS Star catalogue, catalogue No. III/237 at CDS, Strasbourg, at http://vizier.u-strasbg.fr/viz-bin/VizieR?-source=BIS

Mickaelian, A. M., \& Sargsyan, L. A. 2004, Ap, 47, 213

Mickaelian, A. M., Sargsyan, L. A., Erastova, L. K., et al. 2005, Maps of the Cosmos, held in Sydney, July 2003, ed. M. Colless, L. Staveley-Smith, \& R. Stathakis (San-Francisco: ASP), Proc. IAU Symp 216, 230

Monet, D. G., Levine, S. E., Casian, B., et al. 2003, AJ, 125, 984

Moshir, M., Kopan, G., Conrow, T., et al. 1990, Infrared Astronomical Satellite Catalogs, The Faint Source Catalog, Version 2.0, NASA

Olnon, F. M., \& Raimond, E. 1986, IRAS Catalogues and Atlases, Atlas of LowResolution Spectra, A\&AS, 65, 605

Van der Veen, W. E. C. J., \& Habing, H. J. 1988, A\&A, 194, 125

Volk, K., \& Cohen, M. 1989, AJ, 98, 931

Volk, K., \& Kwok, S., Stencel, R. E., \& Brugel, E. 1991, ApJS, 77, 607

Walker, \& H. J., Cohen, M. 1988, AJ, 95, 1801 